\title{
The Structural and Biochemical Characterization of UNC119B Cargo Binding and Release Mechanisms
}

\author{
Tamas Yelland, Esther Garcia, Youhani Samarakoon, and Shehab Ismail*
}

Cite This: Biochemistry 2021, 60, 1952-1963

Read Online

Supporting Information

ABSTRACT: Two paralogs of the guanine dissociation inhibitorlike solubilizing factors UNC119, UNC119A and UNC119B, are present in the human genome. UNC119 binds to N-myristoylated proteins and masks the hydrophobic lipid from the hydrophilic cytosol, facilitating trafficking between different membranes. Two classes of UNC119 cargo proteins have been classified: low affinity cargoes, released by the Arf-like proteins ARL2 and ARL3, and high affinity cargoes, which are specifically released by ARL3 and trafficked to either the primary cilium or the immunological synapse. The UNC119 homologues have reported differences in functionality, but the structural and biochemical bases for these differences are unknown. Using myristoylated peptide binding and release assays, we show that peptides sharing the previously identified UNC119A high affinity motif show significant variations of binding affinities to UNC119B of up to 427-fold. Furthermore, we solve the first two crystal structures of UNC119B, one in complex with the high affinity cargo peptide of LCK and a second one in complex with the release factor ARL3. Using these novel structures, we identify a stretch of negatively charged amino acids unique to UNC119B that may undergo a conformational change following binding of a release factor which we propose as an additional release mechanism specific to UNC119B.

$\mathrm{T}$ he structurally related guanine dissociation inhibitor-like solubilizing factors (GSFs) uncoordinated-119 (UNC119) and phosphodiesterase 6 delta (PDE6D) proteins are encoded by a supergene family and share a beta sandwich immunoglobulin fold. ${ }^{1-3}$ The fold forms a hydrophobic pocket that can accommodate and sequester different lipid modifications (e.g., myristoylated and prenylated proteins) that are present on several membrane-associated proteins. ${ }^{4,5}$ This structural feature allows GSFs to function as solubilizing factors facilitating the diffusion of their otherwise lipophilic cognate cargoes in the cytosol and avoids long residence times on membranes. ${ }^{6,7}$

Two paralogs of UNC119 (UNC119A and UNC119B) have been identified, and both bind and solubilize $\mathrm{N}$-myristoylated proteins. $^{4,8}$ Two classes of UNC119 cargo proteins have been characterized: low affinity cargoes, which are released by the release factors $\mathrm{ADP}$ ribosylation factor-like protein 2 and 3 (ARL2 and ARL3), and high affinity cargoes, which are released specifically by ARL3. ${ }^{8}$ As the guanine nucleotide exchange factor (GEF) for ARL3 and ARL13B ${ }^{9}$ is localized at either the primary cilium ${ }^{10}$ or the immunological synapse, ${ }^{11}$ it has been proposed that high affinity cargoes are trafficked specifically to either of these cellular locations. Low affinity cargoes, however, are proposed to be released by ARL2 (which has low enough affinities for GTP/GDP to facilitate fast nucleotide exchange independent of a GEF) outside the primary cilium and the immunological synapse and are trafficked to other cellular membranes including the plasma membrane and endomembranes.?

UNC119A and UNC119B share 59\% sequence identity and have reported similar cargo specificities in vitro. ${ }^{12}$ Nevertheless, in cells, several functional differences between the two homologues have been observed. Knocking down UNC119B, and not UNC119A, impaired trafficking of myristoylated nephrocystin-3 (NPHP3) to the primary cilium, ${ }^{13}$ while in zebrafish knocking down of UNC119A resulted in a more pronounced ciliary defective phenotype, including a curved body axis, disruption of the ciliary Kupffer vesicle, and visual impairment, than knocking down UNC119B (in zebrafish, UNC119A is homologous to human UNC119B, while UNC119B is homologous to human UNC119A). ${ }^{13}$ Finally, UNC119A mutations have been reported in a cone dystrophy patient $^{14}$ and a separate UNC119A mutation has been reported in a lymphopenia patient. ${ }^{15}$

Received: April 13, 2021

Revised: $\quad$ May 25, 2021

Published: June 15, 2021 
This difference in cellular phenotype raises the possibility that the two UNC119 homologues have uncharacterized functional differences; however, without structural information for UNC119B, it is challenging to propose novel biochemical studies to probe this hypothesis further.

The size of the three amino acids following the $\mathrm{N}$ myristoylated glycine plays a pivotal role in determining the affinity and release of cargo proteins from UNC119A (UNC119A Specific Cargo-USC motif). ${ }^{11,12}$ Using fluorescently labeled peptides conforming to the UNC119A USC motif, we show significant variations in affinity of up to 427fold to UNC119B but only 16-fold to UNC119A. Furthermore, a general pattern of more efficient release from UNC119B than UNC119A is also observed. The cargo peptides include well characterized UNC119 high affinity cargo proteins: NPHP3 (nephrocystin-3 is a protein required for normal ciliary development and is required for renal tubular development), ${ }^{16}$ LCK (lymphocyte kinase-a tryrosine kinase required for $\mathrm{T}$-cell antigen receptor signal transduction at the immunological synapse), ${ }^{17}$ GNAT1 (guanine nucleotide-binding protein $\mathrm{G}(\mathrm{t})$ subunit alpha- 1 , a known cilliary protein alpha subunit heterotrimeric GTPase involved in photoreceptor signaling), ${ }^{18}$ and FMNL1 (formin-like protein 1, a formin involved in regulating actin filament dynamics and shown to localize to the immunological synapse). ${ }^{19}$ An additional four proteins without any reported UNC119 interaction were selected to include as wide a range of biological functions as possible in our analysis: GNAI2 (guanine nucleotide-binding protein $\mathrm{G}(\mathrm{i})$ subunit alpha-2, an alpha subunit heterotrimeric GTPase involved in adenylate cyclase signaling and reported to be N-myristoylated), ${ }^{20}$ PKA (protein kinase A, a cAMP dependent protein kinase with reported cilliary activity ${ }^{21}$ and known to be N-myristoylated), ${ }^{22}$ NSMF (NMDA receptor synaptonuclear signaling and neuronal migration factor, couples NMDA glutamine receptor signaling to the nucleus and is known to be $\mathrm{N}$-mysistoylated), ${ }^{23}$ and PCMTD1 (protein-L-isoaspartate $\mathrm{O}$-methyltransferase domain-containing protein $1-\mathrm{a}$ poorly characterized methyltransferase that is reported to be $\mathrm{N}$-myristoylated). ${ }^{24}$ As these proteins have such a wide range of functions, understanding the intricacies of UNC119 mediated trafficking can improve our understanding of both primary cilia and immunological synapse signaling.

To structurally characterize UNC119B, we solved the first two crystal structures of UNC119B. The first UNC119B structure is in complex with a cargo peptide for the N-terminus of LCK and the second in complex with the release factor ARL3-GppNHp. Using these novel structures and the available ARL2:UNC119A structure (PDB: 4GOK), we propose that a highly negatively charged sequence (residues 120-135) specific to UNC119B may provide an additional release mechanism from UNC119B. This indicates a potential new and different specificity for protein sorting of UNC119B cargoes compared to UNC119A.

\section{MATERIALS AND METHODS}

Cloning. Full-length His-tagged UNC119A and UNC119B constructs were codon optimized for $E$. coli expression as gBlocks from IDT (https://eu.idtdna.com/pages) and cloned into the $\mathrm{pET} 20 \mathrm{~b}$ vector between the $\mathrm{NdeI}$ and $\mathrm{XhoI}$ restriction sites in frame with the C-terminal $\mathrm{His}_{6}$ tag. The UNC119B $\Delta 66$ construct used for crystallization was cloned using the fulllength codon optimized UNC119B as a template using the primers 5'-GGAATTCCATATGGACACCATCCGGCCC-
GAG and 5'-CCGCTCGAGGCCGCTGCCCTGGCCTCCATTATAGGCATAATCAGC. All UNC119 constructs contained a cloning artifact of two additional residues (LE) between the C-terminal UNC119 residue and the $\mathrm{His}_{6}$ tag.

Protein Expression and Purification. C-Terminally noncleavable $\mathrm{His}_{6}$-tagged ARL2 and ARL3 constructs were used from previously published data. ${ }^{11}$ All constructs were expressed and purified using the same protocol with ARL2 and ARL3 buffers supplemented with $5 \mathrm{mM} \mathrm{MgCl}$. Following a fresh transformation into E. coli strain BL21, DE3 (pLysS) cells were grown to O. $\mathrm{D}_{600} \sim 0.6$ and induced with $0.25 \mathrm{mM}$ IPTG and left to express overnight at $18{ }^{\circ} \mathrm{C}$. Once harvested, cells were lysed using a microfluidizer in a buffer containing $40 \mathrm{mM}$ Tris $\mathrm{pH} 7.5,300 \mathrm{mM} \mathrm{NaCl}$, and $2 \mathrm{mM}$ BME. Clarified cell lysate was loaded onto a $5 \mathrm{~mL}$ HisTrap column and washed with lysis buffer supplemented with $20 \mathrm{mM}$ imidazol. Protein was eluted using a linear gradient of $0-300 \mathrm{mM}$ imidazole and fraction content assessed using SDS-PAGE gels. Pooled fractions were concentrated and loaded onto a Superdex S75 column washed in $20 \mathrm{mM}$ Tris $\mathrm{pH} 7.5,150 \mathrm{mM} \mathrm{NaCl}$, and $2 \mathrm{mM}$ DTT. All proteins were snap frozen in liquid nitrogen for storage.

Crystallization and Structure Determination. ARL3 was nucleotide exchanged overnight at $16{ }^{\circ} \mathrm{C}$ using alkaline phosphatase in the presence of a $10 \times$ molar excess of GppNHp (JenaBioscience). Following nucleotide exchange excess, ARL3-GppNHp was incubated with UNC119B $\Delta 66$ and complex separated from excess monomeric ARL3-GppNHp using a Superdex S75 size exclusion column equilibrated in a buffer containing $5 \mathrm{mM}$ Tris $\mathrm{pH} 7.5,20 \mathrm{mM} \mathrm{NaCl}, 4 \mathrm{mM}$ $\mathrm{MgCl}_{2}$, and $2 \mathrm{mM}$ DTT. Small crystals, $<50 \mu \mathrm{m}$, grew in JenaBioscience Classics II screen condition G10 (1.00 M ammonium dihydrogen phosphate, $0.1 \mathrm{M}$ Tris $\mathrm{pH}$ 7.5) within 3 days at $6{ }^{\circ} \mathrm{C}$ at a protein concentration of $8 \mathrm{mg} \mathrm{mL}$. Crystals were snap frozen in liquid nitrogen using the mother liquor solution supplemented with $12 \%$ glycerol and $12 \%$ ethylene glycol. Data was collected at the Diamond Light Source beamline I04. Data was processed automatically with XiaDials and the structure phased using Phaser ${ }^{25}$ with PDB $4 \mathrm{GOK}$ used as a molecular replacement model. Iterative rounds of model building and refinement were performed using $\mathrm{COOT}^{26}$ and REFMAC5 ${ }^{27}$ of the CCP4 program suite ${ }^{28}$ and Phenix.refine. ${ }^{29}$ For the crystallization of the UNC119B $\Delta 66$ :peptide structure, frozen UNC119B $\Delta 66$ was passed over a Superdex S75 size exclusion column equilibrated in a buffer containing $5 \mathrm{mM}$ Tris $\mathrm{pH} 7.5,20 \mathrm{mM} \mathrm{NaCl}$, and 2 $\mathrm{mM}$ DTT, to ensure no aggregates were present. Crystals of UNC119B $\Delta 66$ were obtained by mixing protein with LCK peptide (sequence Myr-GCGCSSHPED-OH, JPT peptide technologies) at a 1:1.1 ratio. Small crystals, $<50 \mu \mathrm{m}$, were obtained in the JBS Classics I screen condition C11 (30\% w/v PEG 4000, 0.1 M HEPES pH 7.5, 0.2 $\mathrm{M} \mathrm{CaCl}_{2}$ ) at $18{ }^{\circ} \mathrm{C}$ at 5 $\mathrm{mg} \mathrm{mL} \mathrm{m}^{-1}$ and grew within 10-14 days. Crystals were flash frozen in liquid nitrogen with the mother liquor solution supplemented with $25 \%$ ethylene glycol.

Fluorescence Polarization. All fluorescence polarization experiments were performed using a Tecan Spark plate reader and Corning half area flat bottom black 96-well plates. Fluorescence was measured with an excitation $\lambda$ at $485 \mathrm{~nm}$ and emission $\lambda$ at $530 \mathrm{~nm}$. All experiments were performed in a buffer containing $5 \mathrm{mM} \mathrm{MgCl}, 20 \mathrm{mM}$ Tris $\mathrm{pH} 7.5,150 \mathrm{mM}$ $\mathrm{NaCl}$, and $2 \mathrm{mM}$ DTT. Fluorescent peptides (FMNL1 - MyrGNAAGSAEQPAGK-FAM, LCK - Myr-GCGCSSHPEDKFAM, NPHP3 - Myr-GTASSLVSPAGGK-FAM, PCMTD1 - 
A

\begin{tabular}{|c|c|c|c|c|c|c|c|c|c|}
\hline Protein & \multicolumn{10}{|c|}{ Sequence } \\
\hline & 1 & 2 & 3 & 4 & 5 & 6 & 7 & 8 & 9 \\
\hline NPHP3 & Myr & G & T & A & S & S & L & V & S \\
\hline GNAT1 & Myr & G & A & G & A & S & A & E & E \\
\hline LCK & Myr & G & C & G & C & S & S & H & P \\
\hline PKA & Myr & G & N & A & A & A & A & K & K \\
\hline NSMF & Myr & G & A & A & A & S & R & R & R \\
\hline FMNL1 & Myr & G & N & A & A & G & S & A & E \\
\hline PCMTD1 & Myr & G & G & A & V & S & A & G & E \\
\hline GNAI2 & Myr & G & C & T & V & S & A & E & D \\
\hline
\end{tabular}

J

\begin{tabular}{|l|c|c|c|}
\hline \multirow{2}{*}{ Peptide } & \multicolumn{3}{|c|}{ Affinity Kd (nM) } \\
\cline { 2 - 4 } & Replicate 1 & Replicate 2 & Average \\
\hline LCK & $1.17 \pm 0.29$ & $0.48 \pm 0.17$ & 0.83 \\
\hline NPHP3 & $0.16 \pm 0.10$ & $1.51 \pm 0.47$ & 0.84 \\
\hline GNAT1 & $1.22 \pm 0.40$ & $4.44 \pm 1.07$ & 2.83 \\
\hline FMNL1 & $1.89 \pm 0.32$ & $5.20 \pm 1.34$ & 3.55 \\
\hline PCMTD1 & $2.03 \pm 0.26$ & $8.44 \pm 1.07$ & 5.24 \\
\hline NMSF & $5.62 \pm 0.90$ & $6.50 \pm 1.12$ & 6.06 \\
\hline PKA & $8.97 \pm 2.12$ & $14.33 \pm 3.94$ & 11.65 \\
\hline GNAI2 & $19.31 \pm 5.53$ & $6.85 \pm 2.31$ & 13.08 \\
\hline
\end{tabular}

\begin{tabular}{|l|c|c|c|}
\hline Peptide & \multicolumn{3}{|c|}{ Affinity Kd (nM) } \\
\hline & UNC119B \\
\hline LCK & Replicate 1 & Replicate 2 & Average \\
\hline NPHP3 & $0.67 \pm 0.22$ & $0.07 \pm 0.09$ & 0.37 \\
\hline GNAT1 & $0.15 \pm 0.05$ & $0.18 \pm 0.07$ & 0.17 \\
\hline FMNL1 & $26.11 \pm 5.38$ & $0.45 \pm 0.45$ & 0.43 \\
\hline PCMTD1 & $35.97 \pm 3.97$ & $109.20 \pm 2.69$ & $\mathbf{7 2 . 5 9}$ \\
\hline NMSF & $2.45 \pm 0.50$ & $2.60 \pm 0.51$ & 2.53 \\
\hline PKA & $11.17 \pm 3.30$ & $12.64 \pm 3.67$ & 11.91 \\
\hline GNAI2 & $25.69 \pm 12.93$ & $29.30 \pm 17.23$ & 27.45 \\
\hline
\end{tabular}

B

LCK

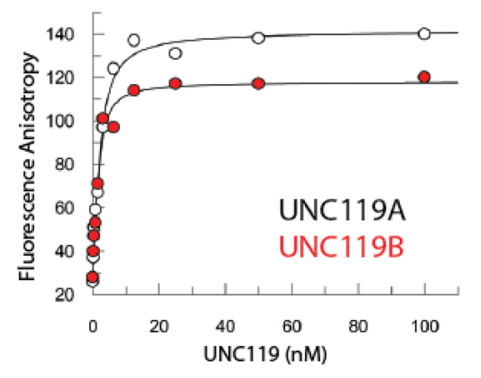

D

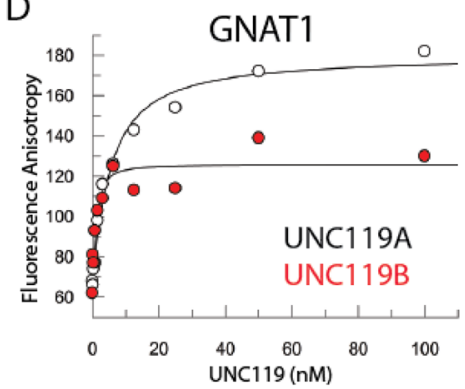

$\mathrm{F}$

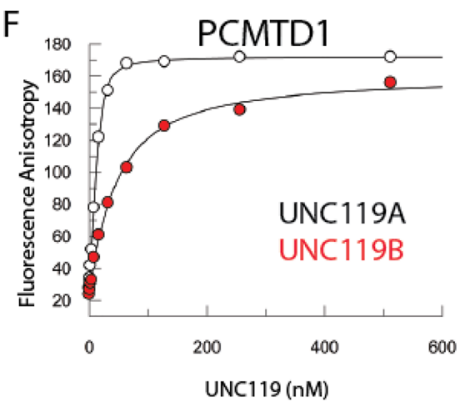

$\mathrm{H}$

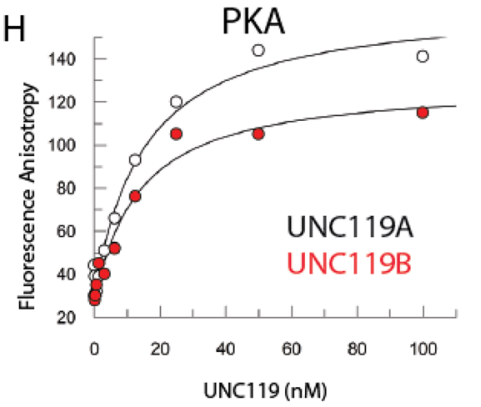

C

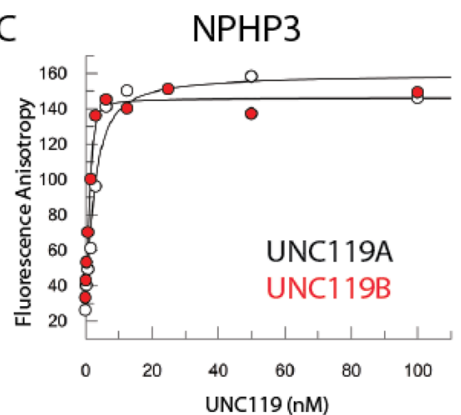

E

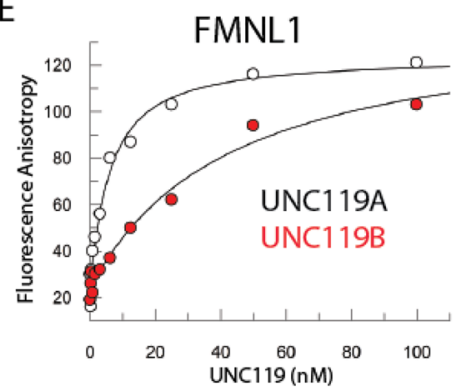

G
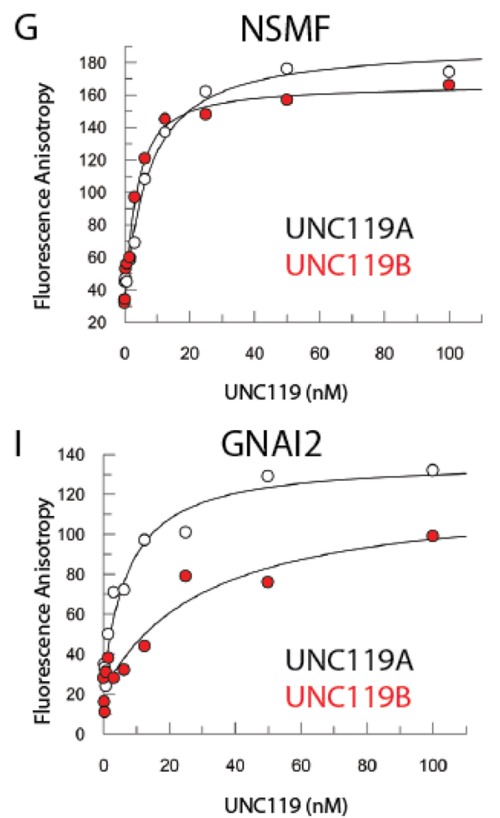

Figure 1. Characterization of peptide binding to UNC119A and UNC119B. (A) Table of theoretical UNC119 high affinity cargo proteins conforming to the USC motif (small residues in positions 3, 4, and 5) highlighted in blue text; the myristoylated glycine residue is shown in red text. Cargoes previously characterized as high affinity for both UNC119A and UNC119B are highlighted in red with newly identified cargoes that bind to UNC119A and UNC119B with comparable affinities in pale red. Cargo proteins characterized as high affinity for UNC119A but not UNC119B are highlighted in green with newly identified cargoes in pale green. Only the first eight residues of each cargo are shown which encompasses the USC motif of theoretical UNC119 high affinity cargo proteins. (B-I) Representative binding curves from one of two independent experiments for LCK, NPHP3, GNAT1, FMNL1, PCMTD1, NSMF, PKA, and GNAI2 to both full-length UNC119A and UNC119B. For all measurements but PCMTD1, the peptide concentration was at $2 \mathrm{nM}$ with UNC119 concentrations ranging from 0.2 to $100 \mathrm{nM}$. For PCMTD1, the peptide concentration was $20 \mathrm{nM}$ with UNC119 concentrations ranging from 1 to $512 \mathrm{nM}$. Data fit using Grafit. (J) Table of the measured binding affinities of peptides to UNC119A and UNC119B.

Myr-GGAVSAGEDNDDK-FAM, PKA - Myr-GNAAAAKKGK-FAM, GNAT - Myr-GAGASAEEKHSRK-FAM, GNAI2 - Myr-GCTVSAEDK-FAM, NSMF - Myr-GAAASRRRAKFAM) were synthesized to greater than $95 \%$ purity and purchased from AltaBioscience. With the exception of PCMTD1, peptide affinity measurements were performed using $2 \mathrm{nM}$ peptide and serial dilution of UNC119A/B from 0.2 to $100 \mathrm{nM}$ with 2-fold serial dilutions. For PCMTD1, a peptide concentration of $20 \mathrm{nM}$ was used and UNC119A/B concentrations from 1 to $512 \mathrm{nM}$ with 2-fold serial dilutions. Peptide release assays were performed using $100 \mathrm{nM}$ peptide, $200 \mathrm{nM}$ UNC119A/B, and $2 \mu \mathrm{M}$ ARL2/3. To obtain dissociation constants, the fluorescence polarization binding data was fitted to a quadratic equation using GraFit: $\mathrm{FP}=F_{\min }$ $-\left(F_{\text {min }}-F_{\text {max }}\right)\left(E+L+K_{\mathrm{d}}-\sqrt{\left(E+L+K_{\mathrm{D}}\right)^{2}-4 E L}\right) / 2 E$, where FP is the fluorescence polarization, $F_{\min }$ and $F_{\max }$ are the minimum and maximum polarization signals, $L$ is the protein 
concentration, $K_{\mathrm{d}}$ is the dissociation constant, and $E$ is the concentration of the fluorescently labeled peptides.

Cell Culture. NIH-3T3 fibroblasts (CRL-1658) were obtained from the ATCC (Manassas, Virginia, US) and cultured following the provider's guidelines, using Dulbecco's Modified Eagle Medium (DMEM) (Gibco, Thermo Fisher Scientific Inc., Waltham, MA, USA) supplemented with $10 \%$ calf serum (ATCC), $2 \mathrm{mM}$ L-glutamine and 100 units $/ \mathrm{mL}$ of penicillin-0.1 mg/mL streptomycin (Sigma, Merck KGaA, Darmstadt, Germany). Jurkat clone E6-1 (TIB-152, human male) from the ATCC was cultured following the provider's guidelines in RMPI 1640 (Gibco, Thermo Fisher Scientific Inc.) supplemented with $10 \%$ inactivated fetal bovine serum (Sigma), $2 \mathrm{mM}$ L-glutamine, 100 units $/ \mathrm{mL}$ of penicillin-0.1 $\mathrm{mg} / \mathrm{mL}$ streptomycin, $1 \mathrm{mM}$ sodium pyruvate, and $10 \mathrm{mM}$ HEPES (both from Sigma). Raji B cells (human, male) were kindly gifted by Dr Pedro Roda-Navarro (Universidad Complutense de Madrid, Spain) and cultured in RMPI 1640 supplemented with $10 \%$ inactivated fetal bovine serum, $2 \mathrm{mM}$ L-glutamine, and 100 units $/ \mathrm{mL}$ of penicillin $-0.1 \mathrm{mg} / \mathrm{mL}$ streptomycin. All cell lines were grown at $37{ }^{\circ} \mathrm{C}$ and $5 \% \mathrm{CO}_{2}$.

Immunofluorescence. For primary cilia labeling, NIH3T3 cells were plated at $80 \%$ confluence on sterile glass 1.5 thick coverslips (VWR, Avantor, Radnor, PA, US) and let to attach overnight for $24 \mathrm{~h}$. Next, cells were starved with $0.5 \%$ calf serum containing media for $24 \mathrm{~h}$ and fixed with $4 \%$ paraformaldehyde (Thermo Fisher Scientific). For staining of the immunological synapse, $1 \times 10^{6}$ cells $/ \mathrm{mL}$ of Raji B cells were incubated with $10 \mu \mathrm{M}$ cell tracker blue (Thermo Fisher Scientific) and $1 \mu \mathrm{g}$ of staphylococcal E enterotoxin (SEE) (Toxin Technologies, Sarasota, FL, USA) for $1 \mathrm{~h}$ at $37^{\circ} \mathrm{C}$. Raji $B$ cells were then washed three times in complete medium by centrifugation. Jurkat $\mathrm{T}$ cells were then added to Raji B cells at a ratio of $1: 1$ and plated onto $0.01 \%$ poly-L-lysine (Sigma) coated coverslips, at approximately $1 \times 10^{5}$ cells per coverslip and incubated at $37{ }^{\circ} \mathrm{C}$ for $20 \mathrm{~min}$ and fixed with $4 \%$ paraformaldehyde for $20 \mathrm{~min}$.

After fixation, cells were extensively washed with PBS and permeabilized using $0.3 \%$ Triton X-100 (Sigma/Merck) for NIH-3T3 or $0.05 \%$ Triton X-100 for Jurkat:Raji conjugates for 5-10 min at room temperature. After thorough washing in PBS, cells were incubated with $2 \%$ BSA (Sigma/Merck) for at least $30 \mathrm{~min}$, followed by primary antibodies (anti-NSMF HPA044316; antiacetylated tubulin MABT868, both from Sigma; anti-PCMTD1 NBP2-55851 from Novus Biologicals, Centennial, CO, USA; and anti-Y394-LCK ab201567 from Abcam, Cambridge, UK), overnight at $4{ }^{\circ} \mathrm{C}$. After extensive washing in PBS, cells were incubated with secondary antibodies (goat antirabbit IgG H+L Alexa Fluor 546 from Thermo Fisher Scientific and goat antimouse IgG $\mathrm{H}+\mathrm{L}$ Abberior Star Red from Abberior GmbH, Göttingen, Germany) for $45 \mathrm{~min}$ in darkness at room temperature. The coverslips were mounted onto glass slides using FluoromountG (Southern Biotech, Birmingham, AL, USA) and cured for at least $6 \mathrm{~h}$ before imaging.

Imaging. All images were collected using a Zeiss 710 upright confocal microscope (Zeiss, Jena, Germany) equipped with a Plan-Apochromat $63 \times / 1.4$ oil DIC M27 objective lens. $z$-Stack images were acquired using a $1 \mu \mathrm{m}$ optical slice for each channel, with a $z$-step of $0.5 \mu \mathrm{m}$ between slices for images of primary cilia and $0.41 \mu \mathrm{m}$ for images acquired of immunological synapse. All microscopy files were processed using Fiji open source software. ${ }^{30}$

\section{RESULTS}

UNC119A High Affinity Cargoes Bind to UNC119B with a Wide Range of Relative Affinities. We have previously reported a motif present for myristoylated proteins that confers high binding affinity to UNC119A-UNC119A specific cargo-USC ${ }^{11,12}$ that requires a small or medium size amino acid at positions 3, 4, and 5. To explore potential differences in binding and release between UNC119A and UNC119B, we set out to identify new uncharacterized cargoes for comparative studies. We searched the protein database (https://www.uniprot.org/) and identified four proteinseither known or reported to be $\mathrm{N}$-myristoylated with no large residues at position 3,4 , or 5-that conformed to the USC motif for biophysical validation using N-terminally myristoylated peptides between 9 and 13 residues: PCMTD1 (a methyltransferase-13-residue peptide), PKA (cAMP dependent protein kinase-9-residue peptide), GNAI2 (a heterotrimeric GTPase-9-residue peptide), and NSMF (involved in NMDA signaling-9-residue peptide). We also included in our study two additional proteins recently identified as UNC119 cargo proteins: LCK (a tyrosine kinase-13-residue peptide) and FMNL1 (a formin regulating actin dynamics-13-residue peptide), ${ }^{11}$ which have had their binding and release specificity characterized in the context of UNC119A but not UNC119B, and two established cargo proteins NPHP3 (ciliary protein13-residue peptide) and GNAT1 (heterotrimeric GTPase13-residue peptide) which have been shown to be specifically released by ARL3 and not ARL2 from both UNC119A and UNC119B. ${ }^{4,13}$

All cargoes bound to UNC119A and UNC119B with tight binding affinities in the nanomolar range; however, several differences in measured affinity were observed (Figure 1). The binding affinities of peptides to UNC119B varied from 0.17 $\mathrm{nM}$ for NPHP3 (Figure 1C) to $72.59 \mathrm{nM}$ for PCMTD1 (Figure 1F). In comparison, peptides bound to UNC119A with a much narrower range of affinities, binding to LCK with a measured $K_{\mathrm{d}}$ of $0.83 \mathrm{nM}$ (Figure 1B) and PCMTD1 with $5.25 \mathrm{nM}$ (Figure 1F). Furthermore, FMNL1 and PCMTD1 bound to UNC119B with affinities between 9.5- and 13.8-fold lower than UNC119A, respectively (Figure 1E and F). All other peptides bind to UNC119A and UNC119B with comparable affinities, and while some differences are observed (e.g., NPHP3 binds to UNC119B with higher affinity than UNC119A-0.17 $\mathrm{nM}$ for UNC119B and $0.84 \mathrm{nM}$ for UNC119A-Figure 1C), these differences are likely within experimental error.

Taken together, our data provides evidence that, while cargo proteins containing the USC motif do bind to UNC119B with high affinity, there is a large range in their relative binding affinities that may hint to different specificities and trafficking within cells.

Cargo Peptides Are More Efficiently Released from UNC119B than UNC119A. Having identified potential differences in protein binding affinities, we sought to determine whether these differences in binding affinities were also reflected in differences in release by either ARL2-GppNHp or ARL3-GppNHp under saturating conditions, which negates differences in binding affinities of ARL2 (reported $K_{\mathrm{d}}$ of 159 $\mathrm{nM}$ to UNC119A and $216 \mathrm{nM}$ to UNC119B) ${ }^{12}$ or ARL3 (reported $K_{\mathrm{d}}$ of $11 \mathrm{nM}$ to UNC119A and $33 \mathrm{nM}$ to UNC119B) ${ }^{12}$ to the different UNC119 homologues (Figure 2 ). This ensures that differences in release are due to the 
A

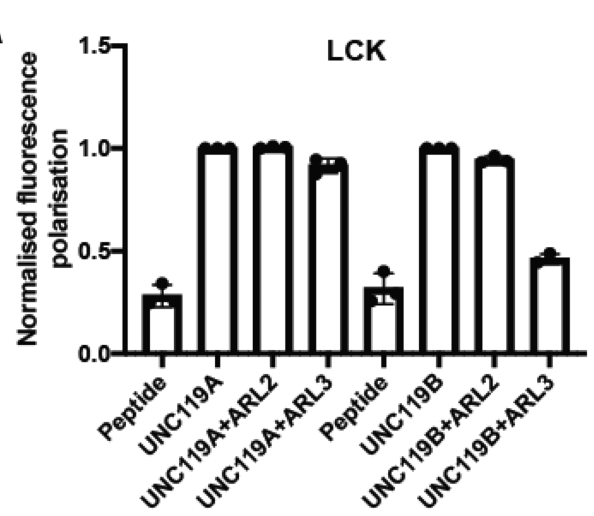

C

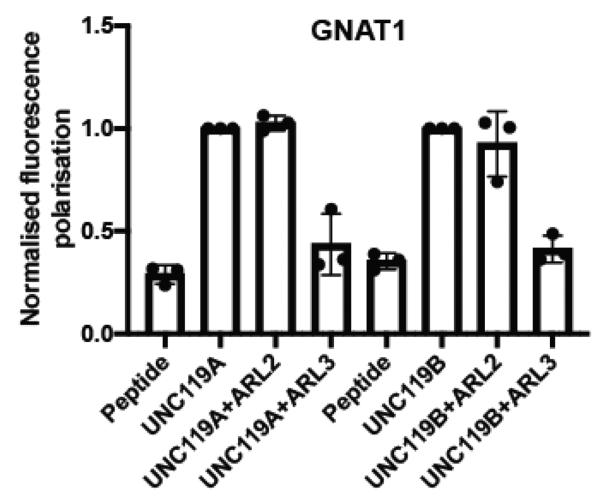

E

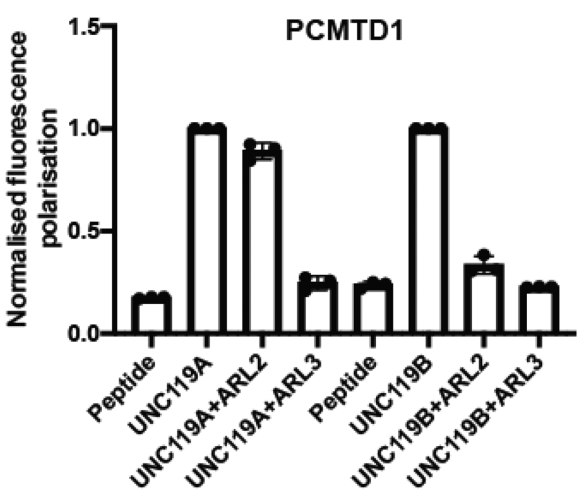

G

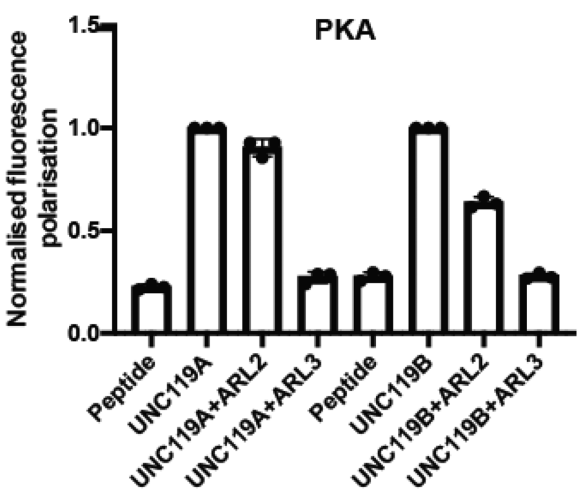

B

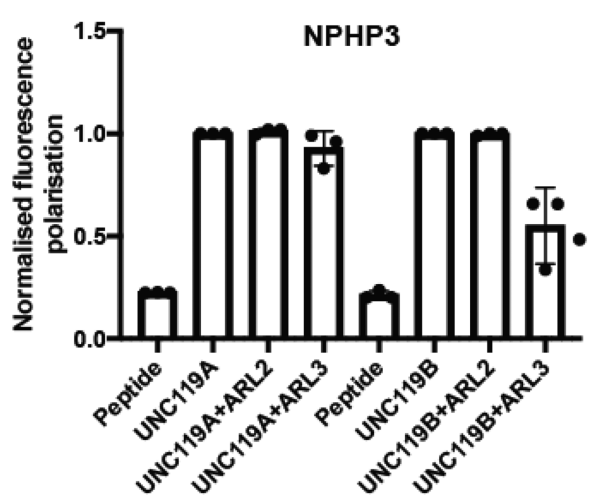

D

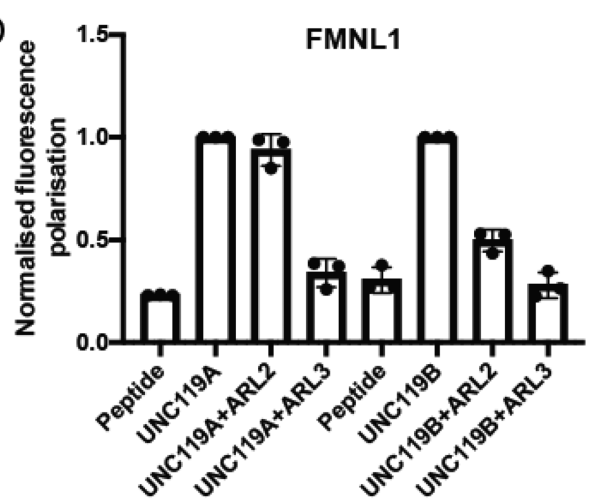

$\mathrm{F}$

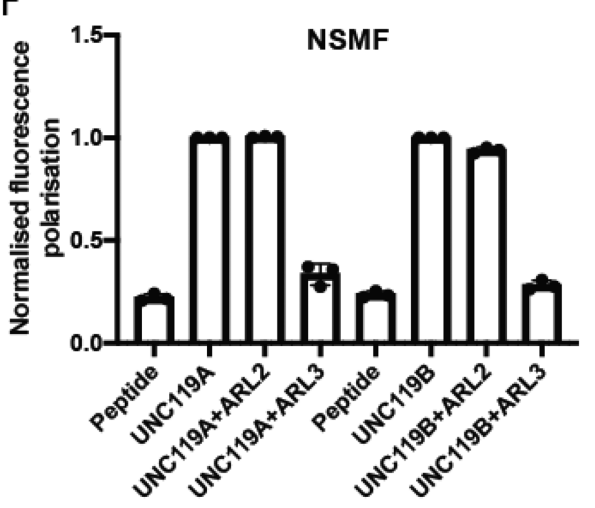

$\mathrm{H}$

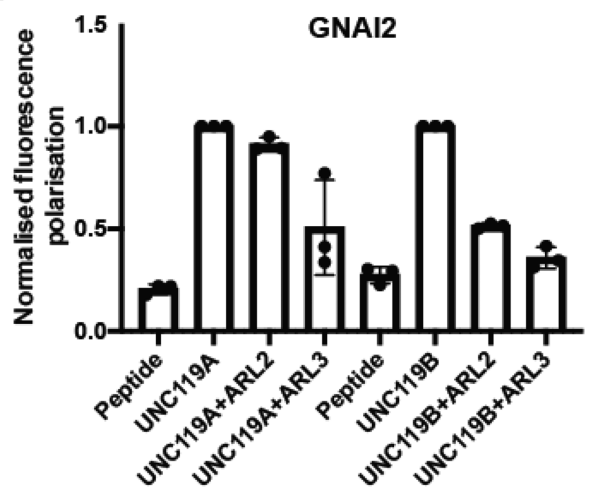

Figure 2. Characterization of peptide release from UNC119A and UNC119B. (A-H) ARL2-GppNHp and ARL3-GppNHp mediated release of fluorescence labeled peptides under saturating conditions. Peptides at $100 \mathrm{nM}$, UNC119 at $200 \mathrm{nM}$, and ARL2-GppNHp and ARL3-GppNHp at 2 $\mu \mathrm{M}$. Charts generated using Prism8. Data shown as mean \pm standard deviation from three independent experiments.

peptide sequence and are not influenced by the fraction of UNC119 bound to either ARL2 or ARL3.
The peptides with the weakest binding affinities to UNC119B (FMNL1, PCMTD1, and GNAI2) are efficiently released by both ARL2 and ARL3 from UNC119B (Figure 
A
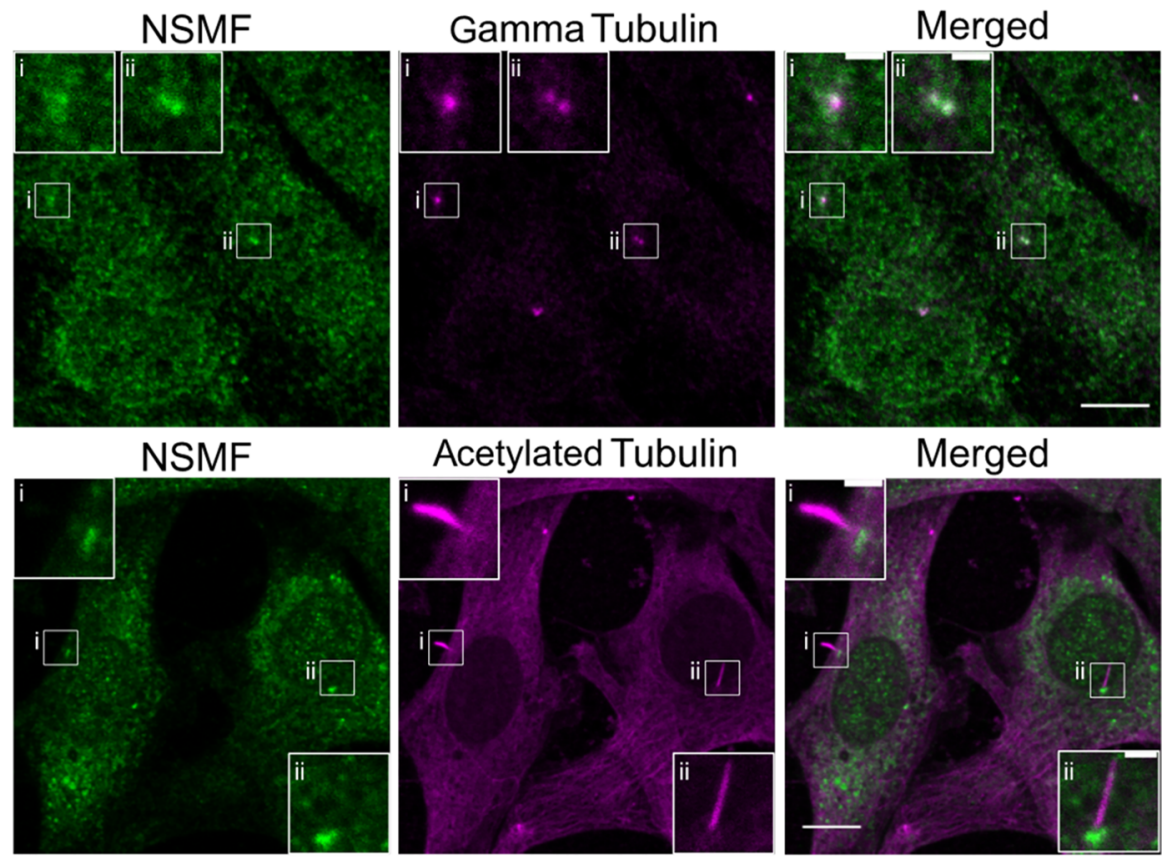

B
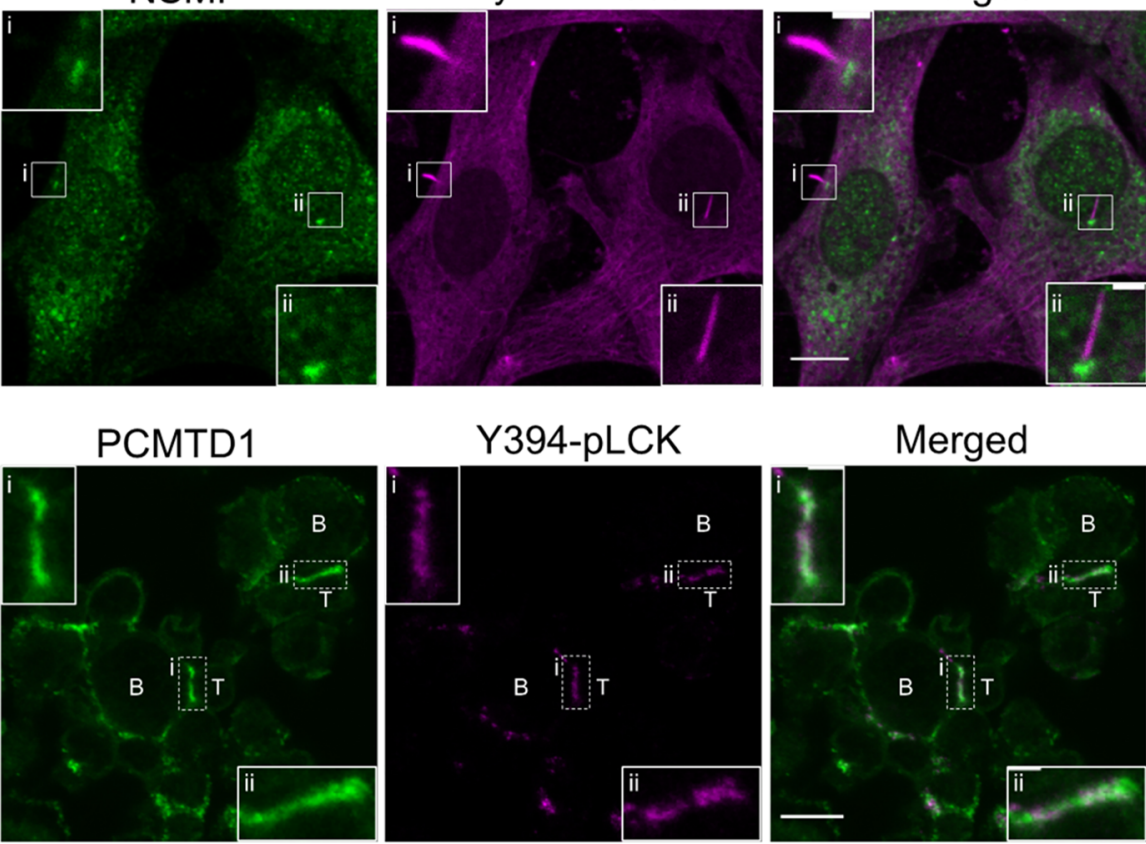

Figure 3. NSMF localizes to the ciliary basal body and PCMTD1 to the immunological synapse. (A) NIH-3T3 cells were grown up to $70-80 \%$ confluence on No. 1.5 glass coverslips and allowed to ciliate in starving media containing $0.5 \%$ fetal bovine serum for $24 \mathrm{~h}$ prior to fixation in $4 \%$ paraformaldehyde. Cells were labeled by immunofluorescence with anti NSMF (green) and either anti gamma (magenta, upper panel) or acetylated tubulin antibodies (magenta, lower panel) showing localization of NMSF to the primary cilia basal body. (B) Jurkat CD4+ T-cells and Raji-B cells (CMAC labeled, not shown) were incubated together in a 1:1 ratio over poly-L-lysine coated No. 1.5 glass coverslips for 10 min prior to fixation with 4\% paraformaldehyde. Jurkat (T):Raji (B) conjugates were immunolabeled with anti PCMTD1 (green) and anti pY394-LCK (magenta) antibodies, showing localization of PCMTD1 to the immunological synapse. Images shown are maximum $z$-projections of three to five optical slices. The scale bars are 10 and $2 \mu \mathrm{m}$ (insets).

2D,E,H). PKA is partially released from UNC119B by ARL2 (Figure 2G) despite comparable binding affinities to both UNC119A and UNC119B. GNAT1 and NMSF show comparable release profiles for both UNC119A and UNC119B where efficient release is only observed following the addition of ARL3 but modest release from UNC119B is observed following the addition of ARL2 when compared to UNC119A; however, these differences are marginal (Figure 2C,F). Finally, the peptides NPHP3 and LCK show more efficient release from UNC119B than UNC119A following the addition of ARL3-GppNHp only (Figure 2A,B). Interestingly, the more efficient release from UNC119B for the LCK peptide occurs despite a higher measured affinity for the peptide $(0.37$ $\mathrm{nM}$ for UNC119B vs $0.83 \mathrm{nM}$ for UNC119A). Although these differences are within experimental error, UNC119B can form additional interactions with the LCK peptide not previously observed in UNC119A (described in more detail below), providing a potential structural explanation for this observation.
In summary, the peptide release profiles correlate well with the measured binding affinities, where lower binding affinity to UNC119B correlates with more efficient release; however, when peptide binding affinities are comparable between UNC119 homologues, more efficient release can still occur in UNC119B, as observed with PKA, NPHP3, and LCK. Finally, regardless of the UNC119 homologue, ARL3 always releases more efficiently than ARL2.

NSMF Localizes to the Ciliary Basal Body and PCMTD1 to the Immunological Synapse. Of the new UNC119 cargoes tested, both PKA and GNAI2 have been shown to have either ciliary ${ }^{31}$ or immunological functions ${ }^{32}$ but, to our knowledge, are not reported as potential UNC119 cargoes. Our new peptide binding and release data suggests UNC119 mediated trafficking may regulate their localization; however, this would require more robust experiments in cells. As PCMTD1 conforms to the high affinity criteria for UNC119A and NSMF to both UNC119A and UNC119B but no reported ciliary or immunological synapse function, we 


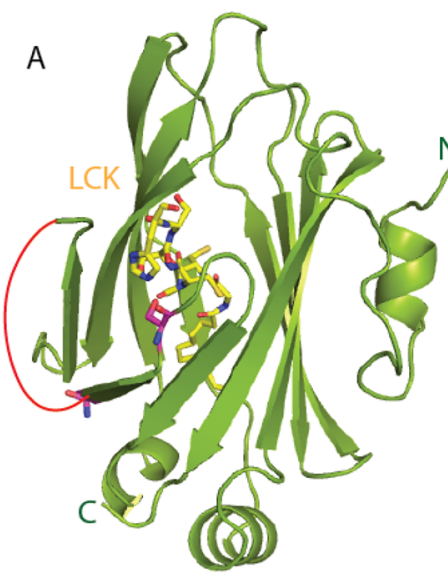

B

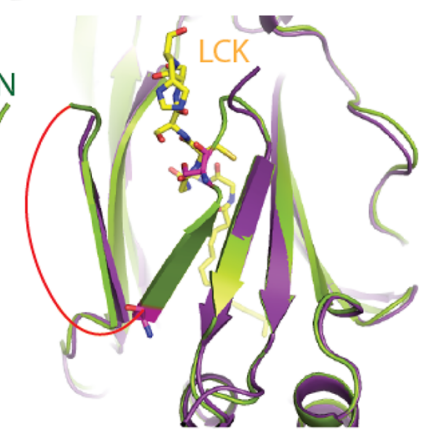

UNC119B Conformation 1 UNC119B Conformation 2
C

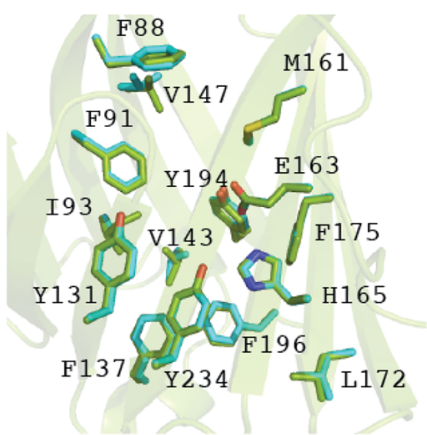

UNC119A
UNC119B

D

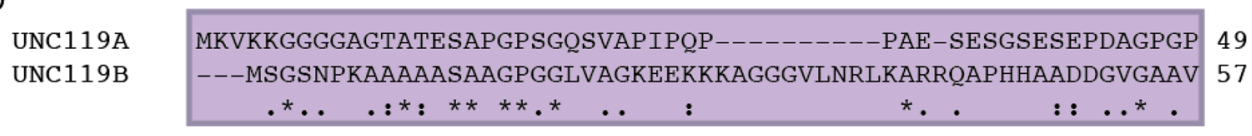

UNC119A RPGPLQRKQPIGPEDVLGLQRITGDYLCSPEENIYKIDFVRFKIRDMDSGTVLFEIKKPP 109 UNC119B TEQELLALDTIRPEHVLRLSRVTENYLCKPEDNIYSIDFTRF KIRDLETGTVLFEIAKPC 117

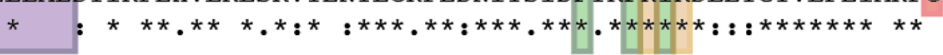

UNC119A VSERLP---INRRDLDPNAGRFVRYOFTPAFLRLROVGATVEFTVGDKPVNNFRMIERHY 166 UNC119B VSDQEEDEEEGGGDVDISAGRFVRYOFTPAFLRLRTVGATVEFTVGDKPVSNFRMIERHY 177 VSDQEEDEEEGGGDVDISAGRFVRYQFTPAFLRLRTVGATVEFTVGDKPVSNFRMIERHY
$* *::$
$\quad \cdot * * * * * * * * * * * * * * * * * * * * * * * * * * * * * * * * * * *$

UNC119A FRNQLLKSFDFHFGFCIPSSKNTCEHIYDFPPLSEELISEMIRHPYETQSDSFYFVDDRL 226 UNC119B FREHLLKNFDFDFGFCIPSSRNTCEHIYEFPQLSEDVIRLMIENPYETRSDSFYFVDNKL 237 $* *:: * * * \cdot * * * \cdot * * * * * * * *: * * * * * * *: * * * * *:: * \quad * * .: * * * *: * * * * * * * *:: *$

$\begin{array}{lll}\text { UNC119A } & \text { VMHNKADYSYSGTP } & 240 \\ \text { UNC119B } & \text { IMHNKADYAYNGGQ } & 251 \\ & : * \star \star * \star * *: * . *\end{array}$

$\mathrm{E}$
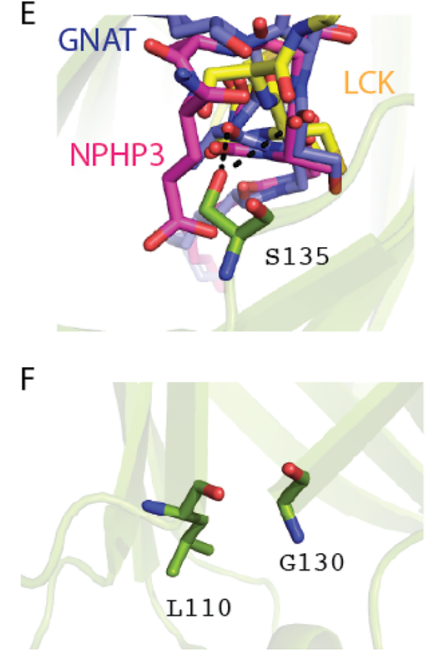

G

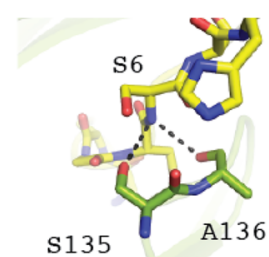

$\mathrm{H}$

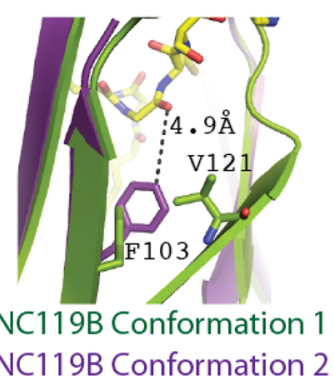

Figure 4. Crystal structure of UNC119B $\Delta 66$ bound to the high affinity LCK peptide. (A) Crystal structure of the UNC119B $\Delta 66: \mathrm{LCK}$ peptide complex. UNC119B is in green cartoon form, while the LCK peptide is in yellow stick form. G130 and S135 are highlighted in pink stick form. The red line connects $\beta$-strands where no interpretable electron density prevents model building. This flexible loop contains the negatively charged acidic loop. (B) Two conformations of UNC119B $\Delta 66$ are observed, one with an additional $\beta$-strand shown in green and the second conformation shown in purple. Bound LCK peptide is shown in yellow stick form. G130 and S135 are highlighted in pink stick form. The red line connects $\beta$ strands where no interpretable electron density prevents model building. (C) Comparison of the UNC119B $\Delta 66$ :LCK complex shown in green with the UNC119A:LCK complex (PDB: 6H6A) shown in blue highlighting the high level of conservation between the two UNC119 homologues. (D) Sequence alignment between human UNC119A and UNC119B. Residues designating the regulatory arm are highlighted in purple. Residues involved in cargo binding are highlighted in green. Residues involved in ARL3 binding are highlighted in yellow. G130 and S135 in UNC119B are not conserved in UNC119A and are highlighted in pink. Residues with no electron density for UNC119B when bound to LCK peptide are highlighted in red and contain the acidic loop. In one chain, the entire loop is disordered with no electron density; in the second, residues G130S135 that form an additional $\beta$-strand can be modeled while the remaining acid residues are disordered. (E) Comparison between the 
Figure 4. continued

UNC119B:LCK (peptide in yellow stick form), UNC119A:NPHP3 (PDB: 5L7K, peptide in pink stick form), and UNC119A:GNAT1 (PDB: 3RBQ peptide in blue stick form) complexes highlights how UNC119B S135 can form steric clashes with NPHP3. An asparagine residue in UNC119A may form mild steric clashes with peptide carbonyl oxygen atoms of NPHP3, GNAT1, and LCK, which could prevent the formation of the additional 2' $\beta$-strand observed in UNC119B. (F) Residue G130 of UNC119B which is not conserved in UNC119A and L110 shown in green stick form with secondary structure shown in cartoon form at $80 \%$ transparency. In UNC119A at the corresponding position is an arginine residue. The large side chain of an arginine would likely form steric clashes with L110 and prevent formation of the additional $\beta$-strand seen in one chain of the UNC119B $\Delta 66$ :LCK peptide co-crystal structure. (G) The $2^{\prime} \beta$-strand forms additional hydrogen bonds (black dashed lines) with the bound LCK peptide through S135 and A136 of UNC119B with the peptide bond of LCK S6. (H) The presence of the additional $2^{\prime} \beta$-strand in UNC119B induces a conformational change in F103 through steric clashes with V121.

sought to determine their cellular localization. Using immunofluorescence, we found that NSMF localizes with gamma tubulin to the ciliary basal body (Figure 3A), while PCMTD1 could be found at the immunological synapse (Figure 3B), together with phosphorylated LCK (Y394), a well-characterized marker of the immunological synapse. This result indicates that these proteins might have new functionality not previously characterized and that their specific localization may be mediated by UNC119 trafficking.

Crystal Structure of UNC119BA66: LCK Peptide Complex Reveals That UNC119B Has a Unique Conformation. To understand the differences in cargo binding and release between the two UNC119 homologues, it is necessary to compare their structures. We solved the crystal structure of UNC119B at $1.95 \AA$ resolution (Supplementary Table 1 and Figure 4A). The protein construct used did not contain the first 66 residues (hereafter called UNC119B $\Delta 66$ ) and was in complex with the Nterminal myristoylated peptide of the high affinity cargo protein LCK (sequence Myr-GCGCSSHPEDD) (Figure 4A). The asymmetric unit contains two copies of UNC119B $\Delta 66$ both in complex with a myristoylated LCK peptide. The two UNC119B $\Delta 66$ chains are not in the same conformation (Figure 4B) with the residues at the mouth of the binding pocket (residues 116-136) either disordered with no interpretable electron density or forming an additional $\beta$ strand ( $\beta$-strand $2^{\prime}-$ Supplementary Figure 1 and Figure 4B) formed through residues 130-135 with residues 116-129 disordered with no interpretable electron density. When comparing to all UNC119A structures (i.e., PDB 5L7K which contains two UNC119A chains within the asymmetric unit, PDB 3RBQ which contains six UNC119A chains within the asymmetric unit, and PDB 6H6A which contains three UNC119A chains within the asymmetric unit), there is never any electron density observed in place of the additional $\beta$ strand of UNC119B, suggesting that this conformation may be unique to UNC119B; however, as the additional $2^{\prime} \beta$-strand is present in only one of the two UNC119B chains, it is possible that, in solution, UNC119B exists in the two forms. Examination of the crystal packing shows that the $2^{\prime} \beta$-strand is not involved in forming crystal contacts (Supplementary Figure 2); however, the loop preceding it is constrained through interactions with a neighboring UNC119B chain (Supplementary Figure 2), which may stabilize the $2^{\prime} \beta$-strand.

We then compared the structures of the UNC119B:LCK complex to the UNC119A:LCK complex (PDB: 6H6A) and confirmed that the residues comprising the lipid-binding pocket are conserved (Figure 4C), which provides a structural explanation as to why binding affinities are similar between UNC119A and UNC119B for LCK. In addition, the conformation of the LCK peptide is the same when bound to either UNC119A or UNC119B (Supplementary Figure 3). Examination of the UNC119 cargo binding pocket highlights why the USC motif (Figure 1A) determines binding affinity to UNC119 proteins: (1) residues 3, 4, and 5 are buried deeply within the UNC119B cargo binding pocket with limited space to accommodate bulky side chains; (2) peptide residues 6 and 7 are located at the opening of the binding pocket where their side chains are solvent exposed, reducing the influence of side chain size at these positions on binding affinity, while residue 8 is fully solvent exposed and forms no interactions with UNC119B. The remaining peptide residues have no interpretable electron density and are likely to be disordered. Unfortunately, the structure does not provide an explanation for why peptides PCMTD1 and GNAI2 bind with significantly lower affinity to UNC119B than UNC119A; the only consistency between the two peptides is a valine residue in position 5 which is slightly larger than all other residues (alanine, serine, and cysteine) in the corresponding position of all of the other peptides. A crystal structure of a UNC119:PCMTD1 complex may be required to definitively answer this specific question.

We compared the conformation of the UNC119B:LCK peptide complex to that of other UNC119A cargo complexes: UNC119A:GNAT1, PDB 3RBQi UNC119A:NPHP3, PDB 5L7K (Figure 4E). The new UNC119B conformation with the additional $2^{\prime} \beta$-strand is unlikely with UNC119A as two residues in UNC119B, G130 (arginine in UNC119A) and S135 (asparagine in UNC119A), are not conserved in UNC119A (residues highlighted in pink, Figure 4D) and would likely disrupt the $2^{\prime} \beta$-strand. The large side chain of an arginine residue would provide significant steric clashes with L110 (Figure 4F), while the asparagine residue in UNC119A would likely form steric clashes with bound NPHP3 and the slightly larger side chain may result in mild steric clashes with the peptide bond carbonyl oxygen atoms which are $2.6 \AA$ from $S 135$, potentially preventing the formation of the $2^{\prime} \beta$-strand observed in UNC119B. This observation leads us to conclude that the presence of the $2^{\prime} \beta$-strand is likely unique to UNC119B. Interestingly, the $2^{\prime} \beta$-strand forms additional interactions with LCK (Figure 4G) through the S135 side chain and A136 peptide bond with LCK S6 and may explain why LCK binds to UNC119B with higher affinity than UNC119A.

The presence of the additional $2^{\prime} \beta$-strand induces changes in the side chain of F103 due to steric clashes with V121 (Figure $4 \mathrm{H}$ ). This new position of F103 is unlikely to have a significant effect on peptide binding, as F103 is $4.9 \AA$ from the nearest LCK peptide residue (Figure $4 \mathrm{H}$ ) and the LCK peptide conformation is largely conserved between the two UNC119B chains. However, the peptide S6 side chain is rotated such that in the UNC119B $\Delta 66$ conformation with the 
A
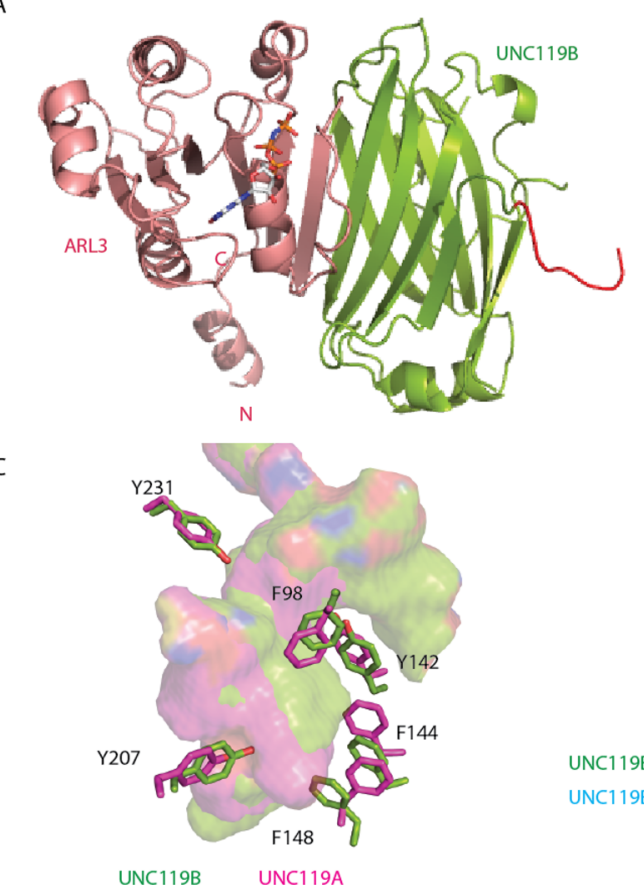

B

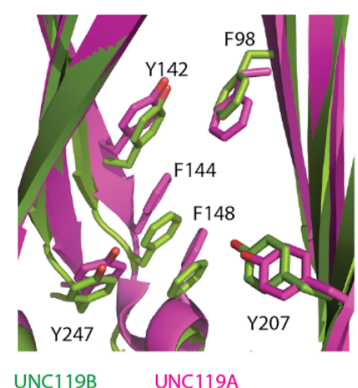

C

Charge selectivity filter sequence UNC119A 107 KPPVSERLP---INRRDLDPNAG 126 UNC119A 107 KPPVSERLP--INR
pI $=6.55$

$\uparrow$

UNC119B 115 KPCVSDQEEDEEEGGGDVDISAG 137

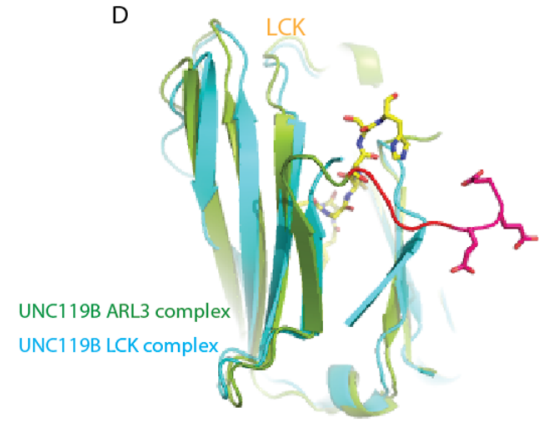

$\mathrm{pI}=3.17$

Figure 5. Crystal structure of the UNC119B $\Delta 66$ :ARL3-GppNHp complex. (A) Crystal structure of the UNC119B $\Delta 66$ :ARL3-GppNHp complex with UNC119B $\Delta 66$ shown in green and ARL3 in salmon. The bound nucleotide GppNHp is in gray stick form. The negatively charged loop at the entrance of the UNC119B cargo binding pocket is colored in red. (B) Comparison of the cargo binding residues of UNC119B (green) with UNC119A (pink) when bound to ARL3. Residues which undergo a shift between the two structures (F98, Y142, F144, F148, Y207, and Y246) are shown in stick form. (C) Residues highlighted in part B (F98, Y142, F144, F148, Y207, and Y246) showing the effect of the residue shift on the cargo binding cavity. (D) The UNC119B $\Delta 66$ :ARL3-GppNHp induces a conformational change placing the residues enriched in glutamic acids in the $2^{\prime} \beta$-strand in proximity of the bound cargo-glutamic acid side chains shown in stick form. UNC119B $\Delta 66$ bound to LCK peptide is shown in blue, while the UNC119B $\Delta 66$ :ARL3 conformation is shown in green. LCK peptide is in yellow in stick form. The negatively charged loop at the entrance of the UNC119B cargo binding pocket is colored in red. (E) Comparison of the residues undergoing a conformational change between UNC119A and UNC119B. UNC119B has a significant enrichment of negatively charged residues resulting in a pI of 3.17 compared to UNC119A which has a $\mathrm{pI}$ of 6.6 for the equivalent residues.

additional $\beta$-strand it faces toward S135 of UNC119B $\Delta 66$. This result is most likely due to the presence of an ethylene glycol molecule in the mouth of the binding pocket than through additional interactions between the two serine side chains.

In summary, the crystal structure of $\mathrm{UNC} 119 \mathrm{~B} \Delta 66$ in complex with the high affinity cargo peptide of LCK reveals that the hydrophobic binding pocket is conserved; however, UNC119B can adopt a novel conformation with an additional $\beta$-strand not previously observed in UNC119A structures that is likely specific to UNC119B.

Crystal Structure of the UNC119BA66: ARL3-GppNHp Complex. As the cargo binding residues are conserved between the two UNC119 homologues, we pursued other avenues to explain differences in cargo release. We solved the crystal structure of UNC119B $\Delta 66$ in complex with the release factor ARL3-GppNHp at $3.15 \AA$ resolution (Figure 5A). The unit cell contains 12 polypeptides and 6 copies of the UNC119B $\Delta 66$ :ARL3-GppNHp complex.

The interface between UNC119B and ARL3 is $\sim 1000 \AA^{2}$ and formed primarily through a parallel $\beta$-strand interaction through switch I of ARL3 and a $\beta$-strand on UNC119B.
Additional electrostatic interactions occur between UNC119B K99 with ARL3 E40 and UNC119B R101 and ARL3 S39 (Supplementary Figure 4A). The interaction between UNC119B and ARL3 is highly conserved when compared to the UNC119A:ARL3 complex (PDB: 4GOJ-Supplementary Figure 4B). This result is to be expected, as the ARL3 binding residues of UNC119B are highly conserved (Figure 1D) with UNC119A and only two residue differences are observed. The first is R198 in UNC119B which is a lysine residue in UNC119A and D206 is a glutamic acid in UNC119A. Neither of these residue differences significantly disrupts surface charge (Supplementary Figure 4C), and in both cases, the side chain is not involved in the interaction. Such conserved interactions help to explain why the binding affinities of UNC119A and UNC119B to ARL3 are so similar (reported 11 and $30 \mathrm{nM}$, respectively). ${ }^{12}$

ARL3 binding to UNC119B induces a conformational change in UNC119B whereby Y194 flips from parallel to perpendicular relative to the myristoyl lipid (Supplementary Figure 5). This results in generating a steric clash with the lipid as well as opening up of the mouth of the binding pocket, reducing the affinity for the cargo and facilitating its release. 
This mechanism is broadly conserved with UNC119A; ${ }^{8}$ however, the aromatic residues which form the myristoyl binding pocket are shifted when compared to the UNC119A:ARL3-GppNHp complex (PDB: 4GOJ) (Figure 5B and $\mathrm{C}$ ), potentially resulting in a greater reduction in cargo affinity than UNC119A; however, this may be influenced by a glycerol molecule from the cryo-protectant present in the binding pocket. While these subtle conformational changes may result in reduced affinity for the cargo in UNC119B relative to UNC119A, it does not explain specific differences in cargo protein release, which are likely to be dependent on the cargo amino acid composition, which would not be affected by the differences in UNC119 lipid binding residue conformation.

One peculiar difference between UNC119B and UNC119A is the presence of a stretch of amino acids (residues 117-135), which is present in the vicinity of the opening of the hydrophobic binding pocket and has a high density of glutamic acid residues producing a strong negative charge at physiological $\mathrm{pH}$ with a $\mathrm{pI}$ of 3.2 (Figure $5 \mathrm{E}$ ). This sequence of residues is not conserved with UNC119A which has a shorter loop, and while charged residues are present with both arginine and aspartic acids residues present, the overall $\mathrm{pI}$ is more neutral at $\sim 6.6$. When comparing the UNC119B peptide bound complex to that bound to ARL3-GppNHp, the $2^{\prime} \beta$ strand at the entrance of the pocket is not present when bound to ARL3 (Figure 5D) and forms interactions with a symmetry mate, and while electron density for the entire loop is not observed, several glutamic acid residues are observed. Without the $2^{\prime} \beta$-strand, this sequence is likely to have increased flexibility in solution and may allow for additional disruptive interactions with the bound cargo peptide. This highly negatively charged sequence could therefore provide an additional release mechanism not found in UNC119A, providing a potential molecular mechanism for the differences detected in cargo binding and release. In line with this hypothesis, the cargo peptides FMNL1, PCMTD1, and GNAI2, which are more efficiently released from UNC119B with ARL2 than UNC119A, all have a negatively charged residue between residues 5 and 10 .

\section{DISCUSSION}

Correct protein cellular localization is key to maintaining normal cellular homeostasis. For soluble proteins, diffusion may be a sufficient mechanism to ensure correct localization. Lipid modified proteins (including many GTPases, kinases, phosphatases, etc.), however, face unique challenges when shuttling between different membrane compartments. The hydrophobic lipid modification needs to be masked from the hydrophilic cytosol in order to maintain protein stability.

UNC119A and UNC119B both bind to N-myristoylated cargoes with affinities in the low nanomolar range depending on the composition of their $\mathrm{N}$-terminal 5 amino acids. ${ }^{11,12}$ Until now, there were no structural or biochemical differences reported despite both homologues having reported different biological functions. It is proposed that ARL3 mediates release of ciliary and immunological synapse ${ }^{11}$ cargoes, whereas ARL2 releases cargoes that localize outside these membrane domains.

As both the primary cilium and the immunological synapse are important signaling hubs, it is necessary to understand their precise protein composition and the mechanisms regulating protein traffic to these systems. Our data provides biochemical evidence that UNC119A and UNC119B have overlapping but not identical cargo specificities (Supplementary Figure 6), which supports previous data of homologue specific trafficking. Our data shows that there can be differences between UNC119A and UNC119B in cargo binding affinities and cargo release most notably in PCMTD1, FMNL1, and GNAI2. Our data provides the first structural comparison between UNC119A and UNC119B and suggests UNC119B cargo affinity and release may be influenced by stretch of negatively charged residues specific to UNC119B at the entrance of the lipid-binding pocket. When bound to a cargo protein, UNC119B can form an additional $\beta$-strand not observed in UNC119A, placing these negatively charged residues away from the cargo protein. When the additional $2^{\prime} \beta$-strand is not present, the negatively charged residues are likely to be flexible in solution. These residues may form additional interactions with the cargo protein in a mechanism similar to that proposed for the N-terminal residues of UNC119A (residues 1-55, termed the "regulatory arm") that have been reported to bind to $\mathrm{LCK}^{11}$ and are proposed to couple UNC119A mediated trafficking with the LCK phosphorylation state. Interestingly, the residues comprising the "regulatory arm" are poorly conserved between UNC119 homologues. It would be intriguing to identify further UNC119 cargo proteins, which can also bind to cargo proteins independently of the lipid in order to identify a "regulatory arm" motif. Due to the significant sequence heterogeneity in the $\mathrm{N}$-terminal residues of UNC119 proteins (Figure 4), such a motif is likely to be highly specific to the different UNC119 homologues and further expand our knowledge on UNC119 homologue specific trafficking.

As all of the peptides bind to UNC119A and UNC119B with high affinity in a cellular context, it is necessary to consider the physiological context of these differences. The primary cilium is a stable structure allowing equilibrium to be reached. In contrast, the immunological synapse is transient, reducing the time to establish equilibrium. As such, differences in protein binding may have a more pronounced effect in the context of the immunological synapse, as it is a more transient structure. Recently, it has been proposed that ARL3 is efficiently activated by its GEF ARL13B (guanine nucleotide exchange factor-a protein that facilitates activation of $\mathrm{g}$ proteins by accelerating nucleotide exchange) only in the presence of another protein, binder of ARL2 (BART) protein which was termed a co-GEF. Furthermore, it was proposed that the ARL3 is kept in its GTP bound, active, form in complex with its co-GEF. ${ }^{33,34}$ It is possible that the interplay between ARL13B, BART, and the ARL3 GTPase activating protein GAP (XRP2) generates a gradient or different local concentrations of ARL3-GTP within the primary cilium and immunological synapse. Both the primary cilium and the immunological synapse are known to be further compartmentalized $^{35,36}$ into specialized microdomains. It is possible that different concentrations of ARL3-GTP could allow for the subtle differences in UNC119 release profiles observed in this study to influence protein trafficking to these microdomains.

\section{ASSOCIATED CONTENT}

\section{(s) Supporting Information}

The Supporting Information is available free of charge at https://pubs.acs.org/doi/10.1021/acs.biochem.1c00251.

Crystallographic table of statistics and figures showing UNC119B structure, crystal packing of the UNC119B $\Delta 66$, comparison of LCK peptide conforma- 
tion between UNC119A and UNC119B, UNC119B $\Delta 66$ ARL3 interaction and cargo release mechanism, and representative electron density (PDF)

\section{Accession Codes}

UNC119A (Q13432), UNC119B (A6NIH7), ARL3 (P36405), and ARL2 (P36404)

\section{AUTHOR INFORMATION}

\section{Corresponding Author}

Shehab Ismail - Beatson Cancer Research UK Institute, Glasgow G61 1BD, Scotland; Institute of Cancer Sciences, University of Glasgow, Glasgow G61 1BD, Scotland; Department of Chemistry, KU Leuven, 3001 Heverlee, Belgium; ๑ orcid.org/0000-0002-4150-1077; Email: shehab.ismail@kuleuven.be

\section{Authors}

Tamas Yelland - Beatson Cancer Research UK Institute, Glasgow G61 1BD, Scotland

Esther Garcia - Beatson Cancer Research UK Institute, Glasgow G61 1BD, Scotland

Youhani Samarakoon - Beatson Cancer Research UK Institute, Glasgow G61 1BD, Scotland

Complete contact information is available at: https://pubs.acs.org/10.1021/acs.biochem.1c00251

\section{Author Contributions}

T.Y. cloned, purified all constructs, obtained crystals, and solved structures. E.G. and Y.S. performed the imaging. All authors contributed to writing.

\section{Funding}

This work was supported by Cancer Research UK core funding number A17196.

\section{Notes}

The authors declare no competing financial interest.

\section{ACKNOWLEDGMENTS}

We thank the Core Services and Advanced Technologies at the Cancer Research UK Beatson Institute with particular thanks to Beatson Advanced Imaging Resource, Biological Services Unit and Molecular Technologies. We thank Diamond Light Source beamline I04-1 for help with data collection.

\section{REFERENCES}

(1) Higashide, T., Murakami, A., McLaren, M. J., and Inana, G. (1996) Cloning of the cDNA for a novel photoreceptor protein. $J$. Biol. Chem. 271 (3), 1797-804.

(2) Hanzal-Bayer, M., Renault, L., Roversi, P., Wittinghofer, A., and Hillig, R. C. (2002) The complex of Arl2-GTP and PDE delta: from structure to function. EMBO J. 21 (9), 2095-106.

(3) Zhang, H., Li, S., Doan, T., Rieke, F., Detwiler, P. B., Frederick, J. M., and Baehr, W. (2007) Deletion of PrBP/delta impedes transport of GRK1 and PDE6 catalytic subunits to photoreceptor outer segments. Proc. Natl. Acad. Sci. U. S. A. 104 (21), 8857-62.

(4) Zhang, H., Constantine, R., Vorobiev, S., Chen, Y., Seetharaman, J., Huang, Y. J., Xiao, R., Montelione, G. T., Gerstner, C. D., Davis, M. W., Inana, G., Whitby, F. G., Jorgensen, E. M., Hill, C. P., Tong, L., and Baehr, W. (2011) UNC119 is required for G protein trafficking in sensory neurons. Nat. Neurosci. 14 (7), 874-80.

(5) Ismail, S. A., Chen, Y. X., Rusinova, A., Chandra, A., Bierbaum, M., Gremer, L., Triola, G., Waldmann, H., Bastiaens, P. I., and Wittinghofer, A. (2011) Arl2-GTP and Arl3-GTP regulate a GDI-like transport system for farnesylated cargo. Nat. Chem. Biol. 7 (12), 9429.

(6) Goody, R. S., Rak, A., and Alexandrov, K. (2005) The structural and mechanistic basis for recycling of Rab proteins between membrane compartments. Cell. Mol. Life Sci. 62 (15), 1657-70.

(7) Schmick, M., Vartak, N., Papke, B., Kovacevic, M., Truxius, D. C., Rossmannek, L., and Bastiaens, P. I. H. (2014) KRas localizes to the plasma membrane by spatial cycles of solubilization, trapping and vesicular transport. Cell 157 (2), 459-471.

(8) Ismail, S. A., Chen, Y. X., Miertzschke, M., Vetter, I. R., Koerner, C., and Wittinghofer, A. (2012) Structural basis for Arl3-specific release of myristoylated ciliary cargo from UNC119. EMBO J. 31 (20), 4085-94.

(9) Gotthardt, K., Lokaj, M., Koerner, C., Falk, N., Gießl, A., and Wittinghofer, A. (2015) A G-protein activation cascade from Arl13B to Arl3 and implications for ciliary targeting of lipidated proteins. eLife 4, No. e11859.

(10) Caspary, T., Larkins, C. E., and Anderson, K. V. (2007) The graded response to Sonic Hedgehog depends on cilia architecture. Dev. Cell 12 (5), 767-78.

(11) Stephen, L. A., ElMaghloob, Y., McIlwraith, M. J., Yelland, T., Castro Sanchez, P., Roda-Navarro, P., and Ismail, S. (2018) The Ciliary Machinery Is Repurposed for $\mathrm{T}$ Cell Immune Synapse Trafficking of LCK. Dev. Cell 47 (1), 122-132.

(12) Jaiswal, M., Fansa, E. K., Kösling, S. K., Mejuch, T., Waldmann, H., and Wittinghofer, A. (2016) Novel Biochemical and Structural Insights into the Interaction of Myristoylated Cargo with Unc119 Protein and Their Release by Arl2/3. J. Biol. Chem. 291 (39), 2076678.

(13) Wright, K. J., Baye, L. M., Olivier-Mason, A., Mukhopadhyay, S., Sang, L., Kwong, M., Wang, W., Pretorius, P. R., Sheffield, V. C., Sengupta, P., Slusarski, D. C., and Jackson, P. K. (2011) An ARL3UNC119-RP2 GTPase cycle targets myristoylated NPHP3 to the primary cilium. Genes Dev. 25 (22), 2347-60.

(14) Kobayashi, A., Higashide, T., Hamasaki, D., Kubota, S., Sakuma, H., An, W., Fujimaki, T., McLaren, M. J., Weleber, R. G., and Inana, G. (2000) HRG4 (UNC119) mutation found in cone-rod dystrophy causes retinal degeneration in a transgenic model. Invest. Ophthalmol. Visual Sci. 41 (11), 3268-77.

(15) Gorska, M. M., and Alam, R. (2012) A mutation in the human Uncoordinated 119 gene impairs TCR signaling and is associated with CD4 lymphopenia. Blood 119 (6), 1399-1406.

(16) Bergmann, C., Fliegauf, M., Brüchle, N. O., Frank, V., Olbrich, H., Kirschner, J., Schermer, B., Schmedding, I., Kispert, A., Kränzlin, B., Nürnberg, G., Becker, C., Grimm, T., Girschick, G., Lynch, S. A., Kelehan, P., Senderek, J., Neuhaus, T. J., Stallmach, T., Zentgraf, H., Nürnberg, P., Gretz, N., Lo, C., Lienkamp, S., Schäfer, T., Walz, G., Benzing, T., Zerres, K., and Omran, H. (2008) Loss of nephrocystin-3 function can cause embryonic lethality, Meckel-Gruber-like syndrome, situs inversus, and renal-hepatic-pancreatic dysplasia. Am. J. Hum. Genet. 82 (4), 959-70.

(17) Barber, E. K., Dasgupta, J. D., Schlossman, S. F., Trevillyan, J. M., and Rudd, C. E. (1989) The CD4 and CD8 antigens are coupled to a protein-tyrosine kinase (p56lck) that phosphorylates the CD3 complex. Proc. Natl. Acad. Sci. U. S. A. 86 (9), 3277-3281.

(18) Calvert, P. D., Strissel, K. J., Schiesser, W. E., Pugh, E. N., Jr, and Arshavsky, V. Y. (2006) Light-driven translocation of signaling proteins in vertebrate photoreceptors. Trends Cell Biol. 16 (11), 5608.

(19) Gomez, T. S., Kumar, K., Medeiros, R. B., Shimizu, Y., Leibson, P. J., and Billadeau, D. D. (2007) Formins regulate the actin-related protein $2 / 3$ complex-independent polarization of the centrosome to the immunological synapse. Immunity 26 (2), 177-90.

(20) Taussig, R., Iñiguez-Lluhi, J. A., and Gilman, A. G. (1993) Inhibition of adenylyl cyclase by Gi alpha. Science 261 (5118), 21821.

(21) Tuson, M., He, M., and Anderson, K. V. (2011) Protein kinase $A$ acts at the basal body of the primary cilium to prevent Gli2 
activation and ventralization of the mouse neural tube. Development 138 (22), 4921-30.

(22) Tholey, A., PipKorn, R., Bossemeyer, D., Kinzel, V., and Reed, J. (2001) Influence of myristoylation, phosphorylation, and deamidation on the structural behaviour of the $\mathrm{N}$-terminus of the catalytic subunit of cAMP-dependent protein kinase. Biochemistry 40 (1), 225-31.

(23) Karpova, A., Mikhaylova, M., Bera, S., Bär, J., Reddy, P. P., Behnisch, T., Rankovic, V., Spilker, C., Bethge, P., Sahin, J., Kaushik, R., Zuschratter, W., Kähne, T., Naumann, M., Gundelfinger, E. D., and Kreutz, M. R. (2013) Encoding and transducing the synaptic or extrasynaptic origin of NMDA receptor signals to the nucleus. Cell 152 (5), 1119-33.

(24) Broncel, M., Serwa, R. A., Ciepla, P., Krause, E., Dallman, M. J., Magee, A. I., and Tate, E. W. (2015) Multifunctional reagents for quantitative proteome-wide analysis of protein modification in human cells and dynamic profiling of protein lipidation during vertebrate development. Angew. Chem., Int. Ed. 54 (20), 5948-51.

(25) McCoy, A. J., Grosse-Kunstleve, R. W., Adams, P. D., Winn, M. D., Storoni, L. C., and Read, R. J. (2007) Phaser crystallographic software. J. Appl. Crystallogr. 40 (4), 658-674.

(26) Emsley, P., and Cowtan, K. (2004) Coot: model-building tools for molecular graphics. Acta Crystallogr., Sect. D: Biol. Crystallogr. 60, 2126-2132.

(27) Murshudov, G., Vagin, A., and Dodson, E. (1997) Refinement of Macromolecular Structures by the Maximum-Likelihood Method. Acta Crystallogr., Sect. D: Biol. Crystallogr. D53, 240-255.

(28) Winn, M. D., Ballard, C. C., Cowtan, K. D., Dodson, E. J., Emsley, P., Evans, P. R., Keegan, R. M., Krissinel, E. B., Leslie, A. G., McCoy, A., McNicholas, S. J., Murshudov, G. N., Pannu, N. S., Potterton, E. A., Powell, H. R., Read, R. J., Vagin, A., and Wilson, K. S. (2011) Overview of the CCP4 suite and current developments. Acta Crystallogr., Sect. D: Biol. Crystallogr. 67, 235-42.

(29) Afonine, P. V., Grosse-Kunstleve, R. W., Echols, N., Headd, J. J., Moriarty, N. W., Mustyakimov, M., Terwilliger, T. C., Urzhumtsev, A., Zwart, P. H., and Adams, P. D. (2012) Towards automated crystallographic structure refinement with phenix.refine. Acta Crystallogr., Sect. D: Biol. Crystallogr. 68 (4), 352-67.

(30) Schindelin, J., Arganda-Carreras, I., Frise, E., Kaynig, V., Longair, M., Pietzsch, T., Preibisch, S., Rueden, C., Saalfeld, S., Schmid, B., Tinevez, J. Y., White, D. J., Hartenstein, V., Eliceiri, K., Tomancak, P., and Cardona, A. (2012) Fiji: an open-source platform for biological-image analysis. Nat. Methods 9 (7), 676-82.

(31) Mick, D. U., Rodrigues, R. B., Leib, R. D., Adams, C. M., Chien, A. S., Gygi, S. P., and Nachury, M. V. (2015) Proteomics of Primary Cilia by Proximity Labeling. Dev. Cell 35 (4), 497-512.

(32) Elgbratt, K., Bjursten, M., Willén, R., Bland, P. W., and Hörnquist, E. H. (2007) Aberrant T-cell ontogeny and defective thymocyte and colonic T-cell chemotactic migration in colitis-prone Galphai2-deficient mice. Immunology 122 (2), 199-209.

(33) Fansa, E. K., and Wittinghofer, A. (2016) Sorting of lipidated cargo by the Arl2/Arl3 system. Small GTPases. 7 (4), 222-230.

(34) ElMaghloob, Y., Sot, B., McIlwraith, M. J., Garcia, E., Yelland, T., and Ismail, S. (2021) ARL3 activation requires the co-GEF BART and effector-mediated turnover. eLife 10, No. e64624.

(35) Blacque, O. E., and Sanders, A. A. (2014) Compartments within a compartment: what $\mathrm{C}$. elegans can tell us about ciliary subdomain composition, biogenesis, function and disease. Organogenesis 10 (1), 126-37.

(36) Krummel, M. F., and Cahalan, M. D. (2010) The immunological synapse: a dynamic platform for local signaling. $J$. Clin. Immunol. 30 (3), 364-72. 